Floating and sinking of self-assembled spheres on liquid-liquid interfaces: rafts versus stacks

Steven G. Jones, ${ }^{1}$ Niki Abbasi, ${ }^{1}$ Abhinav Ahuja, ${ }^{1}$ Vivian Truong, ${ }^{1}$ and Scott S. H. Tsai ${ }^{1, a)}$

Department of Mechanical and Industrial Engineering, Ryerson University, Toronto, Canada M5B $2 K 3$

(Dated: 22 June 2015)

The floating and sinking of objects on fluid-fluid interfaces occurs in nature, and has many important implications in technology. Here, we study the stability of floating self-assembled spheres on an oil-water interface, and how the sphere deposition geometry affects the size limits of the assemblies before they collapse and sink through the interface. Specifically, we compare the critical size of particle rafts to particle stacks. We show that, on liquid-liquid interfaces, monolayer rafts and stacked spheres exhibit different scaling of the critical number of spheres to the Bond number - the dimensionless ratio of buoyancy to interfacial tension effects. Our results indicate that particle stacks will sink with a lower threshold number of particles than particle rafts. This finding may have important implications to engineering applications where interfacial assemblies are not monolayers.

PACS numbers: Valid PACS appear here

Keywords: Floating and sinking, self assembly, interfacial deformation, liquid-liquid interface, hard sphere clusters, Bond number

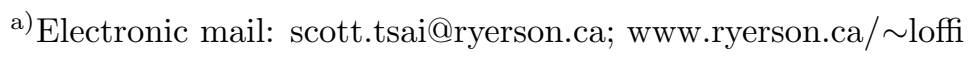




\section{PHYSICS OF FLUIDS}

\section{INTRODUCTION}

The floating and sinking of objects on fluid-fluid interfaces is of practical importance in many applications. ${ }^{1}$ In nature, water striders and other insects depend on the support of surface tension and buoyancy to stand above the free interface of water. ${ }^{2,3}$ Fire ants link their bodies together to improve their collective water repellency, so that they can stay afloat and avoid drowning. ${ }^{4,5}$ In technology, water-walking robots exploit the dependency of the force from surface tension on the robot's surface area, to increase their load carrying capacity. ${ }^{6-8}$ Gravity-induced destabilization of granular rafts and colloid monolayers on fluid-fluid interfaces may be used to encapsulate oils ${ }^{9}$ and create Pickering emulsions, ${ }^{10,11}$ respectively.

In this paper, we consider the self-assembly and destabilization of monodisperse particles at a liquid-liquid interface, and attempt to address the question of how large such assemblies can get before they sink. While related problems have been studied previously in two ${ }^{12-14}$ and three ${ }^{9,15}$ dimensions, those systems only consider monolayer particle rafts. Here, we ask the additional question of what happens when the particle deposition geometry changes; namely, how results change if particles are loaded on top of each other on the liquid-liquid interface (as opposed to particles placed adjacent to each other in forming monolayer rafts).

We first report our experimental findings, then we compare our results to previous calculations of the threshold particle density ratio that identifies collapsible and arbitrarily large rafts and stacks. Finally, for collapsible systems, we develop simple scaling models based on the dimensionless Bond number, for the critical number of spheres that trigger interfacial sinking. We find distinctively different power-laws for particle rafts and stacks, which agree with our experimental observations. Our work also reveals that particle stacks collapse with a lower threshold number of spheres, when compared to particle rafts.

\section{EXPERIMENTAL METHODS}

In experiments, we fill a glass container that has equal side-lengths, $15 \times 15 \times 15 \mathrm{~cm}$, with $2 \mathrm{~L}$ of deionized (DI) water and $1 \mathrm{~L}$ of olive oil (Marque Gallo Brand, Portugal). The DI water and olive oil have densities $\rho_{w}=1,000$ and $\rho_{o}=911 \mathrm{~kg} / \mathrm{m}^{3}$, respectively, and viscosities $\eta_{w}=1$ and $\eta_{o}=84 \mathrm{mPa}$, respectively. Before we deposit spheres on the oil- 
(a)

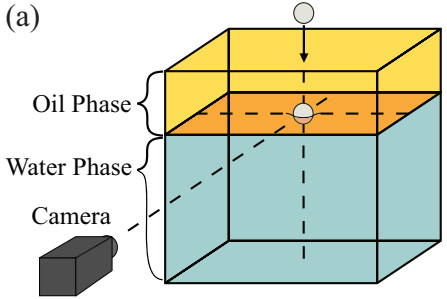

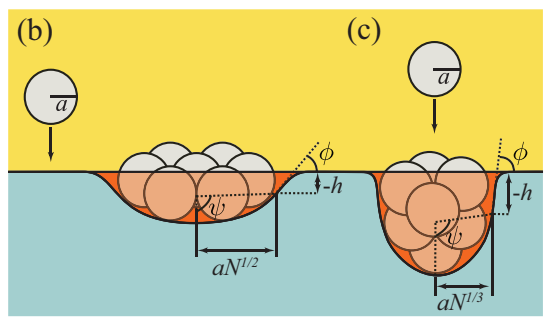

FIG. 1. (Color online) (a) Nylon spheres are deposited, one-at-a-time, from above the glass container. Each sphere descends through the oil phase and settles at the oil-water interface. The spheres attract via a combination of interfacial capillary and buoyancy effects, to form a cluster. The cluster is allowed to completely settle at the liquid-liquid interface before additional spheres are added. (b) A monolayer raft is formed by placing new spheres away from the spheres that are already on the oil-water interface. (c) When spheres are stacked on top of each other, they form an assembly that has an approximate spheroidal shape.

water interface, the oil-water system is allowed to settle for $\sim 2$ hours so that the interface becomes completely stable.

Nylon spheres (Precision Plastic Ball Co., Franklin Park, IL, USA), with density $\rho_{s}=$ $1,150 \mathrm{~kg} / \mathrm{m}^{3}$, and radii $a=1.2$ to $3.2 \mathrm{~mm}$, are deposited from above the oil phase in the container one-at-a-time. The spheres descend through the oil phase and settle on the liquidliquid interface (Fig. 1 (a)). We wait until the self-assembled spheres have reached a stable state before adding more spheres to the assembly. Prior to each experiment the spheres are washed in an isopropyl-based cleaning agent, and air dried.

We modify the interfacial tension of the oil-water interface by adding the surfactant sodium dodecyl sulfate (SDS, Sigma-Aldrich, St. Louis, MO, USA) to the DI water, and measure the interfacial tension using the pendant drop method. We find the oil-water interfacial tensions, $\gamma=11.7$ (4 mM SDS), 14.5 (2 mM SDS), 18.5 (1 mM SDS), and 24.9 (0 $\mathrm{mM} \mathrm{SDS}) \mathrm{mN} / \mathrm{m}$.

We use a digital SLR camera (Nikon D90, Tokyo, Japan) with a marco lens (Nikkor $85 \mathrm{~mm} \mathrm{f} / 3.5 \mathrm{G}$, Tokyo, Japan) to image the spheres' interfacial self-assembly and eventual sinking. The camera is set on a tripod and focused at the center of the oil-water interface (see Fig. 1 (a)). We apply LED back-lighting (Edmund Optics, Barrington, NJ, USA) to improve the image contrast. 


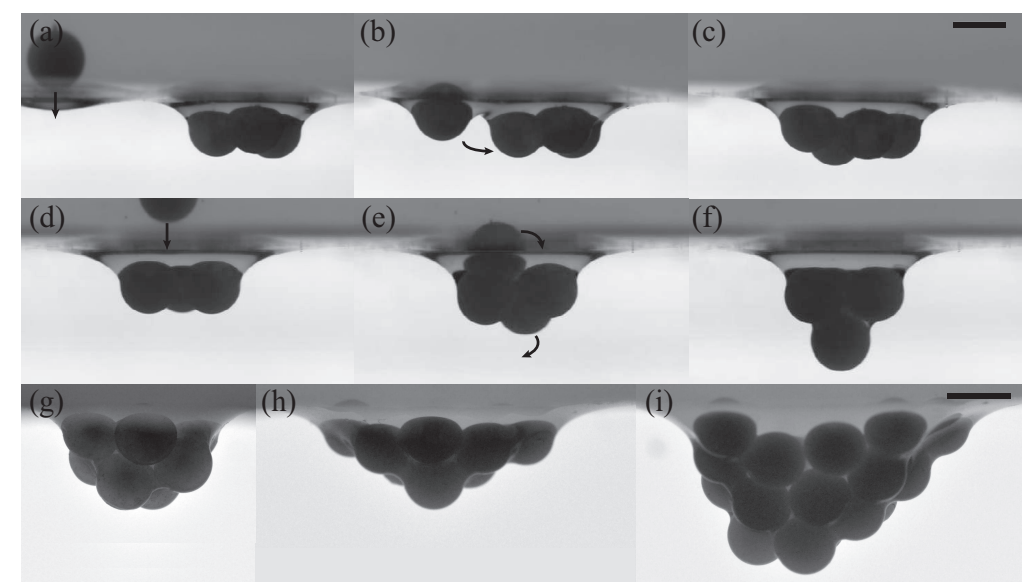

FIG. 2. Spheres of radius $a=2.8 \mathrm{~mm}$, assemble on an oil-water interface with interfacial tension $\gamma=24.9 \mathrm{mN} / \mathrm{m}$. In making interfacial rafts, (a) a sedimenting sphere descends to the liquid-liquid interface away from other spheres, (b) the sphere gets pulled to the particle raft by a combination of interfacial deformation and gravity, and (c) the sphere self-assembles with the other spheres of the existing raft. When we stack the spheres on the liquid-liquid interface, (d) a sphere will descend on top of the spheres that are already at the interface, (e) often resulting in an instability and subsequent cluster rotation, and (f) finally settle into a quasi-equilibrium state at the interface. Large clusters of 10 spheres, each with radius $a=2.2 \mathrm{~mm}$, assemble in equilibrium by the $(\mathrm{g}) \mathrm{raft}$ and (h) stack methods on the oil-water interface. Here, the shape of the equilibrated liquid-liquid interface is much more deformed in supporting a particle stack than a raft of the same number of spheres. The stacked cluster collapses at critical sphere number $N=11$, and (i) the sphere raft supports up to 23 spheres (before collapsing at $N=24$ spheres). Scale bar indicates $5 \mathrm{~mm}$.

\section{RESULTS AND DISCUSSIONS}

\section{A. Assembling interfacial particle rafts and particle stacks}

The two different methods that we use to deposit the spheres result in the formation of sphere rafts (Fig. 1 (b)) and sphere stacks (Fig. 1 (c)). In forming rafts, we place new spheres away from spheres that are already at the interface (Fig. 2 (a)). Depositing new spheres on the oil-water interface causes the spheres to self-assemble by a combination of interfacial deformation and gravity, to form a monolayer on the liquid-liquid interface (Figs. 2 (b) and (c)). This type of interfacial particle self-assembly has been studied previously, ${ }^{16,17}$ 


\section{PHYSICS OF FLUIDS}

and it is termed the Cheerios effect in reference to how cereals tend to cluster on the free interface of a bowl of milk. ${ }^{18}$

In Fig. 2, we differentiate the stacking configuration by the deposition of new spheres on top of existing spheres at the interface (Fig. $2(\mathrm{~d})$ ). Maintaining a new sphere vertically on top of an existing interfacial cluster often results in an instability, where the entire stack will rearrange and rotate until it arrives at a more energetically favorable state (see example Figs. 2 (e) and (f)).

Figs. 2 (g) and (h) show stack and raft clusters, respectively; each cluster has 10 spheres in equilibrium on the liquid-liquid interface. Here the sphere radius $a=2.2 \mathrm{~mm}$, and the liquid-liquid interfacial tension $\gamma=24.9 \mathrm{mN} / \mathrm{m}$. The equilibrated liquid-liquid interface is much more deformed in supporting a particle stack than a raft of the same number of spheres. For stacked sphere clusters, the interface consistently maintains an approximately spheroidal assembly (Fig. $2(\mathrm{~g})$ ); whereas liquid-liquid interfaces that support monolayer particle rafts take on a shape that is more similar to a curved elastic sheet (Fig. 2 (h)). In this particular case, the stack sinks with a critical size $N=11$, while the raft continues to support up to 23 spheres (Fig. $2(\mathrm{i}))$. When the final sphere $(N=24)$ is added to the raft, the raft collapses and passes through the interface.

\section{B. Collapse and sinking at above a critical number of spheres}

Previous calculations ${ }^{13}$ had showed that, in a two-dimensional geometry, where the aspect ratio $t=a / \ell_{c}$ accounts for the finite radius $a$ of the particles and the capillary length $\ell_{c}=\left(\gamma /\left(\rho_{w}-\rho_{o}\right) g\right)^{1 / 2}$, the ratio $D=\left(\rho_{s}-\rho_{o}\right) t /\left(\rho_{w}-\rho_{o}\right)$ determines whether a floating raft can grow arbitrarily large without ever sinking (when $D<D_{\max }$ ), or eventually sink at above a critical particle number (when $D>D_{\max }$ ). Here, $g=9.81 \mathrm{~m} / \mathrm{s}^{2}$ is the acceleration due to gravity, and $D_{\max }$ is the threshold density ratio. The ratio $D$ compares the depth of the interfacial deformation caused by the presence of the particles, to the capillary length $\ell_{c}$.

As described in more detail in a review by Vella, ${ }^{1} D<D_{\max }$ corresponds to the condition where the relative density of the particles is so low that the addition of more particles would simply lower the entire assembly, and the increase in hydrostatic pressure would accommodate the additional particles. When $D>D_{\max }$, the relative density of the particles 


\section{PHYSICS OF FLUIDS}

is sufficiently large that, at a critical number of particles, the aggregate weight from all of the particles lowers the assembly by an amount that is greater than the capillary length $\ell_{c}$, which causes the assembly to sink.

The inset in Fig. 3 (a) shows a plot of our three-dimensional system's threshold density ratio $D_{\max }$ versus the interfacial tension $\gamma \cdot \times$ and + indicates values obtained from experiments of raft and stack configurations, respectively. For each value of interfacial tension $\gamma$, we systematically vary the sphere radius $a$ as we assemble interfacial rafts and stacks. We find that the threshold value $D_{\max } \approx 1$ in all of our three-dimensional raft and stack experiments.

We obtain this result by assuming that we attain an infinite raft or stack when we deplete our supply of (more than 100) spheres, or when the cluster extends to the boundaries of the glass tank, without triggering an instability and sinking. Notably, we observe similar values of the threshold density ratio $D_{\max }$ in both sphere raft and sphere stack experiments. Our experimental value $D_{\max } \approx 1$ is similar to the previous calculation $D_{\max }=\sqrt{2}$, which was made for a two-dimensional geometry, ${ }^{13}$ and our result agrees with Abkarian et al. ${ }^{9}$ who found that axisymmetric monolayer particle rafts do not sink when $D<<1$.

In the regime where $D>D_{\max }$, instability is initiated once the floating raft or stack reaches a critical sphere number, N. Fig. 3 (a) shows a plot of the critical sphere number, $N$, versus individual sphere radius, $a$. Solid symbols show data from raft experiments, and empty symbols reflect results from stacking spheres on the oil-water interface. For each configuration, we also vary the liquid-liquid interfacial tension, $\gamma$, to observe how the interfacial restoring energy affects the critical sphere number $N$. Each data point on the plot is averaged from ten experiments, and error bars represent one standard deviation.

We observe that the critical number of spheres $N$ decreases monotonically with increasing sphere radius $a$, and grows monotonically as the oil-water interfacial tension $\gamma$ is increased. With sphere radius $a$ and interfacial tension $\gamma$ fixed, clusters in the raft configuration tend to grow to a larger sphere number than stacked clusters, before collapsing and sinking through the liquid-liquid interface.

Figs. 3 (b) and (c) show representative image sequences of collapsing rafts and stacks, respectively, immediately after the onset of instability. Here the interfacial tension $\gamma=24.9$ $\mathrm{mN} / \mathrm{m}$, and the sphere radius $a=2.8 \mathrm{~mm}$. The collapse initiates at the location of the critical sphere, and propagates to pull in the surrounding spheres. As the cluster descends 

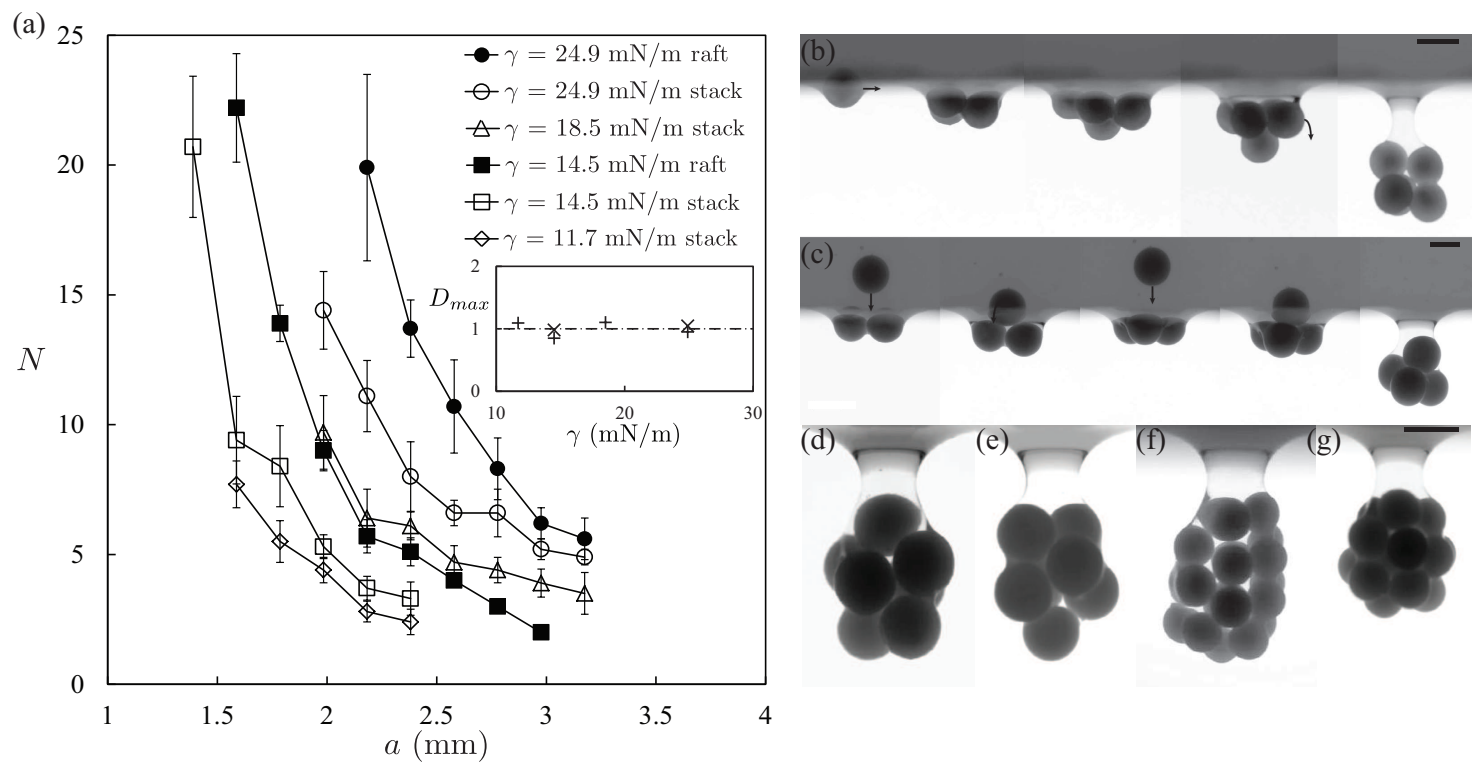

FIG. 3. Sphere rafts and stacks of different sizes are formed on an oil-water interface. The rafts and stacks eventually collapse and sink through the interface when the cluster size reaches a critical sphere number, $N$. (a) The critical sphere number $N$ varies with changes in the sphere radius $a$, the oil-water interfacial tension $\gamma$, and the deposition configuration (either raft or stack). We find that the cluster size $N$ grows monotonically with decreasing sphere size $a$, and with increasing interfacial tension $\gamma$. We also find that spheres deposited into a monolayer raft configuration sinks through the interface as a larger cluster than spheres stacked into a spheroidal stack. Error bars represent one standard deviation. The inset shows the threshold ratio $D_{\max }$ versus interfacial tension $\gamma \cdot \times$ and + indicates values obtained from experiments of raft and stack configurations, respectively. Image sequences of spheres with radius $a=2.8 \mathrm{~mm}$, assembling into (b) rafts and (c) stacks on a liquid-liquid interface, with interfacial tension $\gamma=24.9 \mathrm{mN} / \mathrm{m}$. At above the threshold density ratio $D_{\max }$, both particle rafts and stacks eventually collapse and sink through the liquid-liquid interface. At low sphere numbers, $(N<10)$ both $(\mathrm{d})$ rafts and (e) stacks form similar spheroidal packing arrangements. At larger sphere numbers the packing geometry can differ more significantly between (f) rafts and (g) stacks, although both retain an approximate spheroidal shape. Scale bars represent $5 \mathrm{~mm}$. 


\section{PHYSICS OF FLUIDS}

through the lower water phase, an interfacial neck forms, becomes thinner, and eventually ruptures, while the cluster entrains a thin coating film of the upper oil phase. We anticipate that the coating film thickness will scale with the cluster passage speed, since more rapid passage results in reduced time for the liquid to drain between the cluster and the liquidliquid interface. Additionally, both collapsed rafts (Fig. 3 (d)) and stacks (Fig. 3 (e)) feature the same approximate spheroidal geometry.

Several recent studies have investigated the packing geometry of small hard sphere clusters resulting from energy minimization, ${ }^{19,20}$ such as clusters encapsulated in shrinking droplets. ${ }^{20}$ We observe that in our experiments collapsed sphere clusters often organize into predicted geometries (for example Figs. 3 (d) and (e) form a pentagonal diamond ${ }^{20}$ ), however in some cases the clusters form packing arrangements that are not energetically optimal (for example in Fig. 3 (c) the collapsed cluster with $N=4$ spheres forms a tetrahedron, but in Fig. 3 (b) it does not). We attribute this variation in the cluster geometry to the asymmetrical confinement of the clusters by the liquid-liquid interface during our experiments. For collapsed clusters with $N>10$ spheres, the packing geometry of the spheres can vary considerably, although collapsed clusters still maintain an approximate spheroidal shape (e.g. Figs. 3 (f) and $(\mathrm{g}))$.

\section{Different scaling laws for the critical size of particle rafts and stacks}

To understand at what critical size a particle raft begins to sink, we adapt the generalized equations of Archimedes' principle by Keller, ${ }^{21}$ and mathematical arguments made by Vella et $a l .{ }^{12}$ to our multi-sphere raft geometry (Fig. 1 (b)), such that,

$$
\begin{aligned}
& \frac{4}{3} \pi N g a^{3}\left(\rho_{s}-\rho_{o}\right)=2 \pi \ell_{r} \gamma \sin \psi \sin \phi+ \\
& \quad \pi N g a^{3}\left(\rho_{w}-\rho_{o}\right)\left(-\frac{h}{\ell_{r}} \sin ^{2} \psi+\frac{2}{3}-\cos \psi+\frac{1}{3} \cos ^{3} \psi\right)
\end{aligned}
$$

Here, the particle raft characteristic length $\ell_{r}$, the height of the interfacial inflection line above the undeformed interface $h$, the angle of inclination of the interface at the inflection line $\phi$, and the angular position of the inflection line relative to the center of the particle raft $\psi$ (see Fig. 1). 


\section{PHYSICS OF FLUIDS}

The left side of (1) balances gravity acting on the entire raft to the restoring forces on the right: force from interfacial tension (first term) and buoyancy force (second term). We note that the force from interfacial tension scales with the circumference of the particle raft, and the buoyancy and gravity terms are both based on the raft volume. We have added the critical sphere number $N$ to the gravity and buoyancy terms to account for multiple spheres at the liquid-liquid interface, and we use $\ell_{r}$ to calculate the circumference of the raft. We also assume that the spheres only wet the oil phase, so that a three-phase contact line is not present. This assumption is supported by our observations that, upon destabilization and sinking, the nylon spheres become completely coated with a thin film of oil.

We approximate the raft characteristic length $\ell_{r}$ using the raft coverage area such that $\ell_{r}^{2} \approx N a^{2}$. As a result, the raft characteristic length $\ell_{r} \approx a N^{1 / 2}($ Fig. $1(\mathrm{~b}))$. Then we define the Bond number $B o=\left(\rho_{s}-\rho_{o}\right) g a^{2} / \gamma$, the aspect ratio $H=h / \ell_{r}$, and reorganize (1) to obtain,

$$
N=\alpha B o^{-2}+\beta B o^{-1}
$$

where the $O(1)$ pre-factors,

$$
\alpha=\frac{36 \sin ^{2} \psi \sin ^{2} \phi}{\left(2-4 D / t-3 \cos \psi+\cos ^{3} \psi\right)^{2}} \quad \text { and } \quad \beta=\frac{9 H^{2} \sin ^{4} \psi}{\left(2-4 D / t-3 \cos \psi+\cos \psi^{3}\right)^{2}}
$$

These simplifications result in the scaling relationships, $N \propto B o^{-2}$ when Bond number $B o$ is small (i.e. restoring force dominated by interfacial tension), and $N \propto B o^{-1}$ when Bond number $B o$ is large (i.e. buoyancy effects important).

Fig. 4 (a) shows a log-log plot of the critical sphere number $N$ versus the Bond number Bo in our experiments based on a raft geometry (see inset schematic). By fitting once with $\alpha \approx 3.5$ and $\beta \approx 0.5$ in (2), we find an excellent agreement between the experiments and our model. We note that, over the range of Bond numbers Bo in our experiments, interfacial tension plays a much more significant restoration role than buoyancy effects: the model resembles $N \propto B o^{-2}$ more closely than $N \propto B o^{-1}$ in the scope of our observations. The scaling $N \propto B o^{-2}$ also appears in other related studies of interfacial raft formation where 

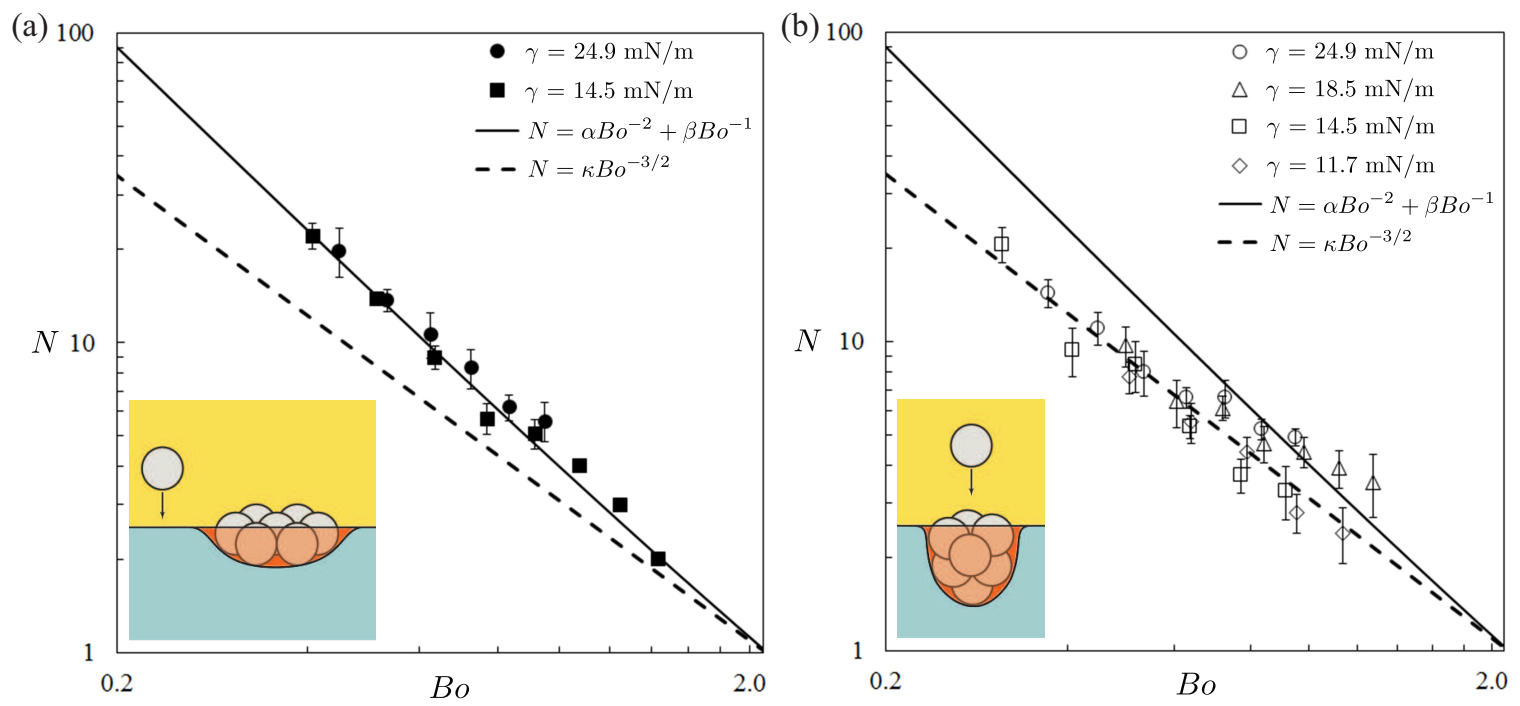

FIG. 4. (Color online) Log-log plots of critical sphere number $N$ versus Bond number $B o$, giving two different power laws for sphere raft and stack configurations. (a) The solid line shows that clusters assembled in a raft configuration sink through the oil-water interface at a critical sphere number $N=\alpha B o^{-2}+\beta B o^{-1}$. (b) The dashed line shows that, when we stack spheres on the oil-water interface, the resulting critical sphere number $N=\kappa B o^{-3 / 2}$.

buoyancy effects is neglected, ${ }^{10,15}$ but our more generalized model (2) will be applicable in higher ranges of the Bond number $B o$, where buoyancy plays a more significant role than interfacial tension.

In the sphere stacking configuration, we use the stack volume $\ell_{s}^{3} \approx N a^{3}$ to extract the stack characteristic length $\ell_{s} \approx a N^{1 / 3}$ (see Fig. 1 (c)). Replacing $\ell_{r}$ in (1) with $\ell_{s}$, and re-arranging as in (2), we obtain the quadratic expression,

$\left(N^{-1 / 3} B o^{-1 / 2}\right)^{2}-\frac{1}{2} \frac{\sin \psi}{\sin \phi} H\left(N^{-1 / 3} B o^{-1 / 2}\right)+\frac{1}{\sin \psi \sin \phi}\left(\frac{1}{6} \cos ^{3} \psi-\frac{1}{2} \cos \psi-\frac{2}{3} \frac{D}{t}+\frac{1}{3}\right)=0$.

The solution to (4) is in the form $N^{-1 / 3} \mathrm{Bo}^{-1 / 2}=\kappa^{-1 / 3}$, where the $O(1)$ pre-factor,

$$
\begin{aligned}
\kappa^{-1 / 3}= & \frac{1}{24} \frac{1}{\sin \psi \sin \phi}\{3 H-3 H \cos 2 \psi+ \\
& \left.2\left[-6(8-16 D / t-9 \cos \psi+\cos 3 \psi) \sin \psi \sin \phi+9 H^{2} \sin ^{4} \psi\right]^{1 / 2}\right\} .
\end{aligned}
$$


Thus, the expression for the stacking geometry's critical sphere number,

$$
N=\kappa B o^{-3 / 2} .
$$

Notably, we find that in the stacking geometry, the critical sphere number, $N \propto B o^{-3 / 2}$, for all values of the Bond number $B o$, regardless of whether interfacial tension or buoyancy dominates the restorative force.

In Fig. $4(\mathrm{~b})$, we calculate $\kappa \approx 3.1$ by ensuring that the stacking geometry's model, $N=\kappa B o^{-3 / 2}$, converges to the raft geometry's expression, $N=\alpha B o^{-2}+\beta B o^{-1}$, when the critical sphere number $N=1$ (since the two modes of sphere deposition must be identical when there is just one sphere). The data from our experiments show a good agreement with the with the stack scaling $N=\kappa B o^{-3 / 2}$.

We note that the stack data is biased towards the raft model at lower values of sphere number $N$, and the deviation is more pronounced in experiments where the interfacial tension is higher; for example, when $\gamma=24.9$ and $18.5 \mathrm{mN} / \mathrm{m}$, as indicated by empty circles and triangles, respectively, in Fig. 4 (b). We attribute this deviation to the tendency of stacks, at low sphere number $(N<4)$, to reorient into a raft configuration (see for example the third frame of Fig. 3 (c)).

We interpret this observation by the following hypothesis. When a new sphere is stacked on top of a forming sphere cluster, it may cause the cluster to rotate to a new equilibrium position, which occurs over time $t_{e q}$. When the critical sphere is added, the cluster accelerates and passes through the oil-water interface over time $t_{\gamma}$. If the ratio of stabilization time $t_{e q}$ to interface passage time $t_{\gamma}$ is greater than unity, $t_{e q} / t_{\gamma}>1$ (i.e. passage occurs quickly) then the cluster will remain in the stack orientation as it passes through the interface. However if $t_{e q} / t_{\gamma}<1$ (i.e. reorientation happens more rapidly than passage), then the spheres will reorient into a raft, $\ell_{s} \rightarrow \ell_{r}$, where $\ell_{r}>\ell_{s}$, consequently increasing the interfacial tension contribution supporting the cluster, and preventing the cluster from sinking through the interface.

This effect diminishes at higher sphere numbers, because there is a greater difference in the critical sphere number between rafts and stacks. At higher sphere numbers, our 


\section{PHYSICS OF FLUIDS}

experimental data clearly shows that there is a distinctively different scaling of the critical sphere number $N$, for particle stacks compared to rafts.

\section{CONCLUSIONS}

These experimental results and scaling analyses show that, at above the threshold density ratio $D_{\max }$, floating self-assembled interfacial rafts and stacks will eventually collapse and sink when they reach a critical size. The scaling dependence of the critical sphere number $N$ on the Bond number $B o$ is distinct between rafts and stacks: particle rafts collapse when the sphere number $N=\alpha B o^{-2}+\beta B o^{-1}$; particle stacks follow the scaling for the sphere number, $N=\kappa B o^{-3 / 2}$.

This different scaling law for particle stacks may have important engineering implications in situations where the floating assemblies on liquid-liquid interfaces are not monolayers. For example, our recent work shows the clustering and coating of self-assembled magnetic microparticles in microfluidics. ${ }^{22}$ Flow-focused microparticles assemble into clusters on the co-flowing interface between water and oil in a multi-layered fashion. Thus, the size control of these microfluidic coated particle clusters may be determined by a power law similar to the one we show for particle stacks - albeit with a magnetic Bond number to account for magnetic forces in the microfluidic system.

\section{ACKNOWLEDGMENTS}

S. S. H. Tsai acknowledges the Natural Sciences and Engineering Research Council (NSERC) of Canada for supporting this project through the Discovery grant program. S. G. Jones acknowledges funding support from the Ontario Graduate Scholarships program. The authors thank I. M. Griffiths for reviewing an early draft of this manuscript.

\section{REFERENCES}

${ }^{1}$ D. Vella, "Floating versus sinking," Annu. Rev. Fluid Mech. 47 (2014).

${ }^{2}$ J. W. M. Bush, D. L. Hu, and M. Prakash, "The integument of water-walking arthropods: form and function," Adv. Insect Physiol. 34, 117-192 (2007). 


\section{PHYSICS OF FLUIDS}

${ }^{3} \mathrm{X}$. Gao and L. Jiang, "Biophysics: water-repellent legs of water striders," Nature 432, 36-36 (2004).

${ }^{4}$ N. J. Mlot, C. A. Tovey, and D. L. Hu, "Fire ants self-assemble into waterproof rafts to survive floods," Proc. Natl. Acad. Sci. U.S.A. 108, 7669-7673 (2011).

${ }^{5}$ P. C. Foster, N. J. Mlot, A. Lin, and D. L. Hu, "Fire ants actively control spacing and orientation within self-assemblages," J. Exp. Biol. 217, 2089-2100 (2014).

${ }^{6}$ L. Wu, Z. Lian, G. Yang, and M. Ceccarelli, "Water dancer II-a: a non-tethered telecontrollable water strider robot," Int. J. Adv. Robotic Sy. 8, 10-17 (2011).

${ }^{7}$ O. Ozcan, H. Wang, J. D. Taylor, and M. Sitti, "Surface tension driven water strider robot using circular footpads," in IEEE Int. Conf. Robot. (IEEE, 2010) pp. 3799-3804.

${ }^{8}$ Y. S. Song and M. Sitti, "Surface-tension-driven biologically inspired water strider robots: Theory and experiments," IEEE Trans. Robot. 23, 578-589 (2007).

${ }^{9}$ M. Abkarian, S. Protière, J. M. Aristoff, and H. A. Stone, "Gravity-induced encapsulation of liquids by destabilization of granular rafts," Nat. Commun. 4, 1895 (2013).

${ }^{10}$ J. W. Tavacoli, G. Katgert, E. G. Kim, M. E. Cates, and P. S. Clegg, "Size limit for particle-stabilized emulsion droplets under gravity," Phys. Rev. Lett. 108, 268306 (2012).

${ }^{11}$ J. W. Tavacoli, J. H. J. Thijssen, and P. S. Clegg, "Particle-stabilized oscillating diver: a self-assembled responsive capsule," Soft Matter 7, 7969-7972 (2011).

${ }^{12}$ D. Vella, D.-G. Lee, and H.-Y. Kim, "The load supported by small floating objects," Langmuir 22, 5979-5981 (2006).

${ }^{13}$ D. Vella, P. D. Metcalfe, and R. J. Whittaker, "Equilibrium conditions for the floating of multiple interfacial objects," J. Fluid Mech. 549, 215-224 (2006).

${ }^{14}$ J.-L. Liu, X.-Q. Feng, and G.-F. Wang, "Buoyant force and sinking conditions of a hydrophobic thin rod floating on water," Phys. Rev. E 76, 066103 (2007).

${ }^{15}$ N. Vandewalle, N. Obara, and G. Lumay, "Mesoscale structures from magnetocapillary self-assembly," Eur. Phys. J. E Soft Matter 36, 1-6 (2013).

${ }^{16}$ E. H. Mansfield, H. R. Sepangi, and E. A. Eastwood, "Equilibrium and mutual attraction or repulsion of objects supported by surface tension," Phil. Trans. R. Soc. A 355, 869-919 (1997).

${ }^{17}$ P. A. Kralchevsky and K. Nagayama, "Capillary interactions between particles bound to interfaces, liquid films and biomembranes," Adv. Colloid Interface Sci. 85, 145-192 (2000).

${ }^{18}$ D. Vella and L. Mahadevan, "The cheerios effect," Am. J. Phys. 73, 817-825 (2005). 


\section{PHYSICS OF FLUIDS}

${ }^{19}$ G. Meng, N. Arkus, M. P. Brenner, and V. N. Manoharan, "The free-energy landscape of clusters of attractive hard spheres," Science 327, 560-563 (2010).

${ }^{20}$ V. N. Manoharan, M. T. Elsesser, and D. J. Pine, "Dense packing and symmetry in small clusters of microspheres," Science 301, 483-487 (2003).

${ }^{21}$ J. B. Keller, "Surface tension force on a partly submerged body," Phys. Fluids 10, 30093010 (1998).

${ }^{22}$ S. S. H. Tsai, J. S. Wexler, J. Wan, and H. A. Stone, "Conformal coating of particles in microchannels by magnetic forcing," Appl. Phys. Lett. 99, 153509 (2011). 